\title{
Handover Comparisons of WiMAX and WiMAX Release 2
}

\author{
Ali Sufyan ${ }^{1}$ \\ GCUF, Sahiwal, Pakistan \\ E-mail: ali_sufyan@ymail.com ${ }^{1}$ \\ Yasir Salam ${ }^{2}$, Sundas Amin ${ }^{3}$ \\ E-mail: yasirsalam08@ hotmail.com², sundusramey@ ciitsahiwal.edu.pk ${ }^{3}$
}

Received: 17 April 2017; Accepted: 05 June 2017; Published: 08 August 2017

\begin{abstract}
Internet has become major need of every person. It provides information in a single click, cost effective way of communication with the outside world, gigantic library, social networking, feeds and blogs, satellite TV and business. World has become a global village because of internet. WiMAX is one of the most promising and innovating technology which supports high speed data and multimedia applications like VoIP, Satellite TV and video conferencing. The demand for enlarged coverage and high speed internet made WiMAX services still inadequate to the users. WiMAX Release 2 or WiMAX 2 is 4G technology built on IEEE $802.16 \mathrm{~m}$ specification that will provide high bandwidth for VoIP and peak download rate up to $300 \mathrm{Mbps}$ which is more than double the current WiMAX. This work enlightens features and comparison of WiMAX and WiMAX 2 with respect to different handover schemes, network architecture, bandwidth, spectral efficiency and sector size throughput. Result shows that the WiMAX 2 is similar to WiMAX but with increased bandwidth, $4.5 \%$ better spectral efficiency and 28/54 Mbps Sector/site throughput.
\end{abstract}

Index Terms - WiMAX, VoIP, 4G, WiMAX 2, Spectral Efficiency, Sector/site Throughput.

\section{INTRODUCTION}

With the rapid growth of internet, greater bandwidth and high data rate connection requirement has led to many advanced and optimized technologies. Express and continuous growth of private networks, e-commerce and bandwidth intensive applications like video conferencing and multimedia raised the demand for higher bandwidth[1].

In recent years there has been growing interest shown in wireless technologies for user access. Although the 802.16 family of standards is authoritatively called WMAN (Wireless Metropolitan Area Network) but also known as "WiMAX" (Worldwide Interoperability for Microwave Access). It offers broadband wireless access that is one of the most promising and innovative technology which made the high speed broadband services possible in a better and economical way as compared to existing cable or DSL, providing the last mile high data rate internet access in remote areas. . The deployment of WiMAX in the world is under process by 475 operators in almost 140 countries[2].

WiMAX has potential to do to broadband internet access which mobile phones have done to communication access. WiMAX has changed the priorities of broadband customers to replace cable and DSL connections in the same way people have renounce land lines in favor of cell phones. The main purpose of this project is to make the WiMAX technology better for future wireless internet[3].

WiMAX interoperability enables to connect fixed and portable mobile users. Moreover it provides invisible connectivity among hot-spots. WiMAX is an IP based, wireless broadband access technology that is quite similar to 802.11 (Wi-Fi) standard but intended for wireless metropolitan area networks (MAN). WiMAX can provide broadband wireless access up to 30 miles for fixed stations and 3 to 10 miles for mobile stations whereas Wi-Fi is limited to only 100 to 300 feet in most cases. WiMAX has adopted the advanced radio features in order to reduce cost and to ensure interoperability. The recent Long Term Evolution (LTE) standard is analogous to WiMAX which is developed as a counterpoint to WiMAX. In addition, WiMAX gave the low cost as compared to $3 \mathrm{G}$, HSPDA, xDSL, HFC etc. Fig.1 is giving an overview of WiMAX deployment with other existing technologies[4].

To improve WiMAX network capacity, vendors are in partnership with WiMAX operators now a days. WiMAX equipment's are cheap and the WiMAX Forum is working to build cost effective and enhanced performance network with an upgrade for the growing demand of broadband services which is named WiMAX 2 and based on IEEE $802.16 \mathrm{~m}$ standard[5]. WiMAX is frequently focused on two most important areas of concern namely location management and handover management[4].

Over the proposed is to contribute the involvement for this intention to study and comparison Handover schemes 
of WiMAX and WiMAX Release 2. It involves three handover techniques at MAC layer for WiMAX network, comparison of network architecture, sector/site throughput (Mbps) and queuing delay of WiMAX and WiMAX Release 2. OPNET is used to simulate handover schemes.

To meet above stated importance of the work, our objectives are 1) to study of WiMAX and WiMAX Release 2. 2) Implementation of handover schemes for Mobile WiMAX at MAC layer. 3) Comparison of sector/site throughput (Mbps), queuing delay and site spectral efficiency of WiMAX and WiMAX Release 2. 3) Collection of results to compare WiMAX and WiMAX Release 2.

\section{RELATED WORK AND MOTIVATION}

Over the last few years the growing demand for mobile broadband and multimedia applications of internet produced a profitable interest between operators to determine latest technologies to offer these services at an affordable cost. A number of vendors and operators from all over the world has joined the IEEE 802.16 (Institute of Electrical and Electronics Engineers) and WiMAX Forum to develop the new demands.

The name WiMAX was created by WiMAX forum formed in June 2001 to develop interoperability and promote conformity. The IEEE 802.16 standard forms the basis of WiMAX and referred to as WiMAX (802.16), Fixed WiMAX (802.16e) and Mobile WiMAX (802.16d). The IEEE 802.16d developed in 2004 and known as a fixed WiMAX but it had no support for mobility[6]. The IEEE 802.16e is an amendment to 802.16-2004 which introduced support for mobility and therefore called "Mobile WiMAX" which is now established in many countries[2].

WiMAX access was used in tsunami in December 2004 to assist with communication in Indonesia when all other communication infrastructures was destroyed making people unable to communicate to outside world or vice versa. At that time WiMAX provided access that helped a lot to restart communication.

WiMAX is based on IEEE 802.16e-2005/IEEE 802.16e-2009 has been commercially deployed since 2006 and in over 140 countries there are more than 500 deployments with worldwide presence and proven performance. Customer demand for broadband data is growing rapidly[3]. To address the capacity and performance constraints the migration path for network enhancements is very much necessary so WiMAX Operators are looking for a timely and cost-effective migration path. Hence they are either now encountering or expect to encounter in the near future[7].

A vertical handoff decision algorithm that can select an optimum target network based on bandwidth requirement, battery power, cost of service, network performance and network condition.

The performance improvements supported by WiMAX 16e-Enhanced can be grouped into three major performance categories[7]: 1) Improved Link Budget in the UL. 2) Enhanced Interference Management to enable a reuse factor of 1.3) An increase in spectral efficiency and system throughput.

$3 \mathrm{G}$ communication is the main opponent of WiMAX such as UMTS and CDMA2000. In addition, existing Wi-Fi and mesh technologies are providing high speed mobile data applications. The evolution of major cellular standard so called $4 \mathrm{G}$ is the real competitor with highbandwidth, low latency and all IP-networks with voice services etc. UMTS is the genuine effort of 3GPP LTE. Fig.1 is showing the speed vs. mobility of wireless systems.

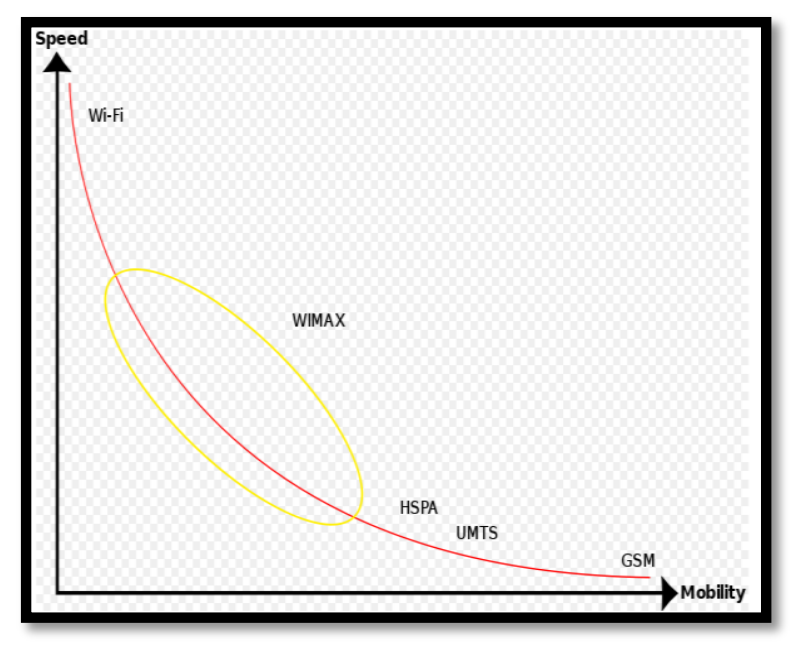

Fig.1. Speed vs. Mobility

\section{Mobile WiMAX Network Architecture:}

Network Reference Model (NRM) represents the main functional entities and reference points over which WiMAX network architecture interoperability framework is defined[8]. It consists of:
1. Mobile Station
2. Access Service Network
3. Connectivity Service Network

\section{A. Mobile Station}

Mobile station (MS) is equipment that provides wireless link between WiMAX network and subscriber station (SS).

It is further divided into three categories[9]:

- Radio equipment's same as mobile handsets that provides link with base stations (BTSs) are called Mobile WiMAX Terminals.

- Portable equipment's such as laptop and PDA are identical to mobile terminal apart from supporting some special applications, called Portable WiMAX Terminals.

- Wireless and fixed devices which are connected to Customer Premises Equipment (CPE) for connectivity with base station, called Fixed WiMAX Terminals. 


\section{B. Access Service Network (ASN)}

The Access Service Network (ASN) represents the entry point of WiMAX MS to MiMAX network as shown in Fig.2. It provides support to a set of network functions which are necessary to offer radio access to the Mobile Station (MS)[10]. It is also called Network Access Provider (NAP). A network access point is a business entity which offers WiMAX radio access infrastructure for one or more than one ASNs. It also provides WiMAX services and IP connectivity to WiMAX users according to agreed and defined service levels agreements (SLAs) with NAPs. To support mobility the following functions are compulsory for ASNs:

- Layer-2 connectivity with a user terminal

- Quality of Service and policy Management

- Radio Resource Management (RRM)

- To establish Layer-3 connectivity with MS

To provide mobility management the Access Service Network (ASN) provides the following functions[2]:

- ASN-anchored mobility

- $\quad$ Paging and Location Management

- CSN-anchored mobility

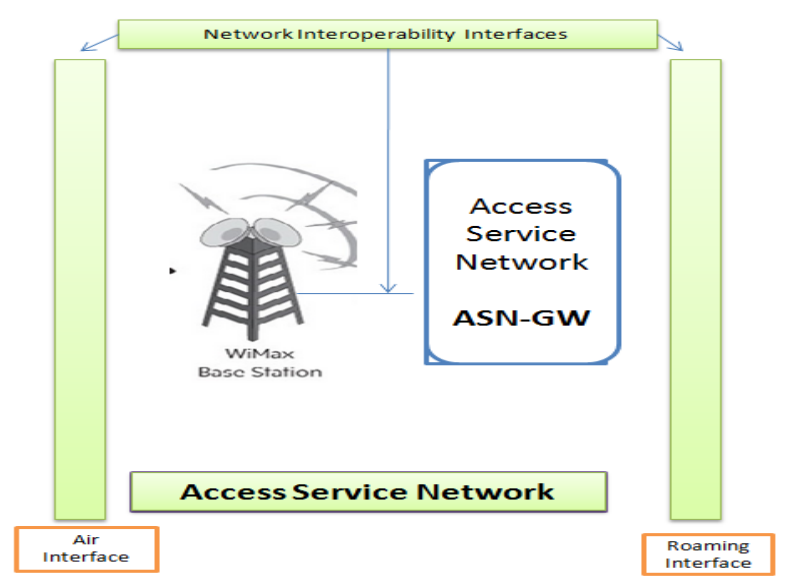

Fig.2. Access Service Network

\section{Connectivity Service Network (CSN)}

The Connectivity Service Network (CSN) consist of network functions which offer IP Services and comprises a number of network entities such as AAA servers, interworking gateway devices and user databases for authentication. It provides following functions:

- $\quad$ Roaming

- AAA Services

- Inter-CSN tunneling for roaming

- Internet Access

- Security Authentication

- Billing and inter-operator agreement

- Inter-ASN mobility

\section{DESIGN METHODOLOGY}

The design scheme of three different handovers of Mobile WiMAX is simulated and designed. The Network models and signaling messages of these scenarios are designed by using Microsoft Office PowerPoint 2013 while OPNET 16 Modular is used to analysis the performance. In OPNET modular handover schemes and their network, node, link and process model of hard handover, micro diversity handover and fast base station switching handover are designed at Media Access Control (MAC) layer.

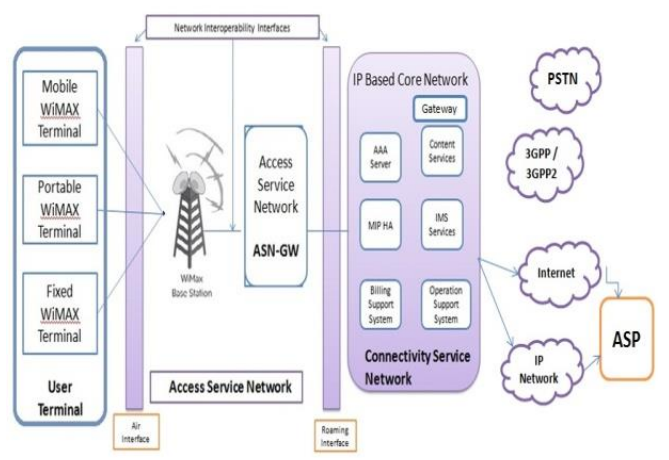

Fig.3. WiMAX Architecture

\section{A. Network Design}

The network model consists of three entities Mobile Station (MS), a Backbone Network and three Base Stations (BSc). Mobile Station (MS) is attached with BS wirelessly to obtain WiMAX service which is further connected with Backbone Network. BS is responsible for transmitting and receiving data to and from either the Mobile Station (MS) or Backbone Network as shown in Fig.4.

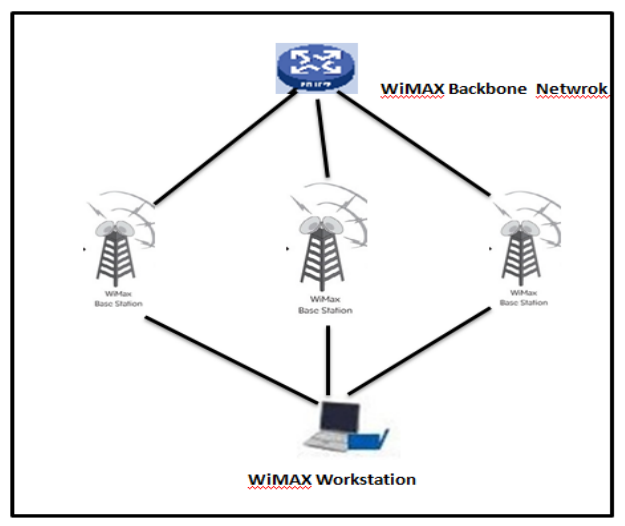

Fig.4. Network Model of WiMAX MAC layer

\section{B. Hard Handover}

The IEEE 802.16.2009 standard clearly defines the hard handover algorithm that is used to design this scenario [15]. There are six types of MAC messages that are used during handover. At the start MS connects with BS1 till the signal level of the BS1 reduces. At this stage mobile station send MOB_MSHO_REQ to the serving BS1 which then send C_HO_REQ to BS2 all the way 
through Backbone network to notify about the MS request[14] .BS2 is a target base station in this situation. It will send C_HO_RSP to BS1[11].

A response message MOB_MSHO_RSP contains information of target BS2 will be send by serving BS1. Mobile station sends a message MOB_HO_IND along with C_HO_IND type to serving Base Station 1 that shows verification of mobile station handover. Finally BS2 will start contact with MS and similar process will be performed when signal strength of BS will decrease to establish handover with BS3.

\section{Fast Base Station Switching (FBSS)}

In Fast Base Station Switching (FBSS) all three BSs are in diversity set during anchor [12] BS updating.

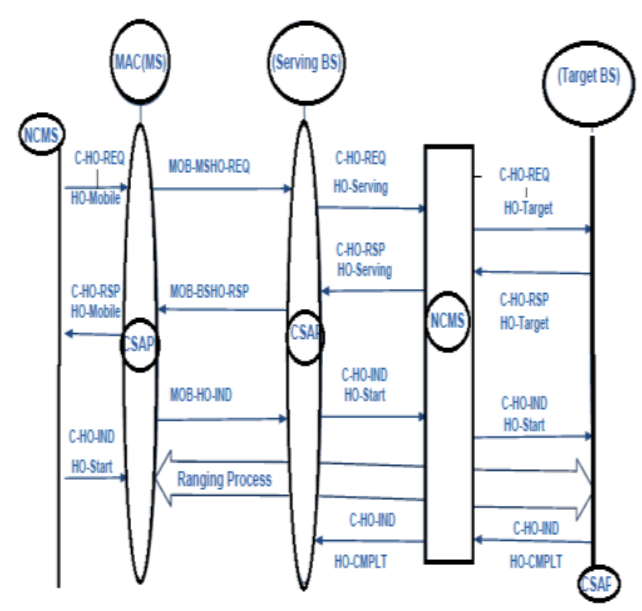

Fig.5. Signaling of target MS and Serving BS

MAC signaling is shown in Fig.5. Firstly Base Station one is anchor BS which is used for uplink and downlink. After defined time Base Station two and three will act as anchor Base Station. Mobile Station selects an anchor BS by sending MOB_MSHO_REQ message to serving anchor BS. At this stage serving BS will send MOB_BSHO_RSP to MS. Mobile Station then either accepts anchor update by sending MOB_HO_IND or rejects by MDHOFBSS_IND_type to 0b01[13].

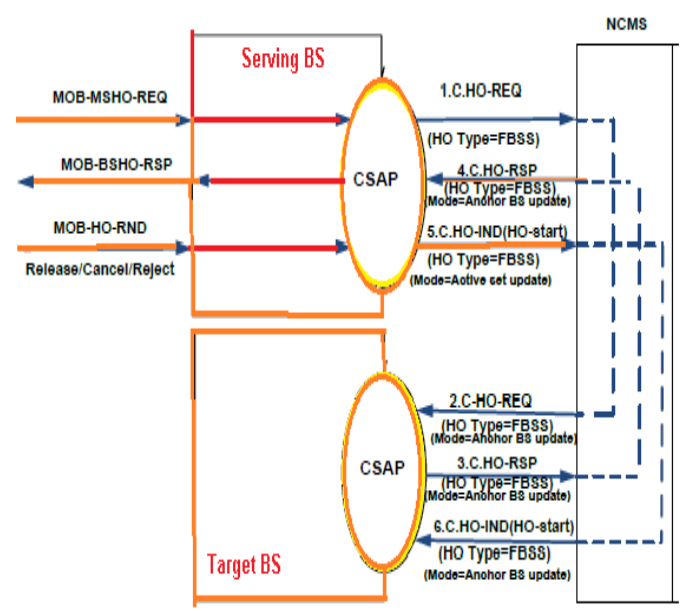

Fig.6. MAC Signaling of FBSS [7]

\section{Micro Diversity Handover (MDHO)}

The IEEE 802.16.2009 algorithms for connecting and disconnecting BS are used in Micro Diversity Handover (MDHO). Initially supposed all three base stations are in diversity set and after some time BS1 disconnected because of low threshold rate and attach to BS2 which will drop after 14400 seconds due to low threshold value and connects to BS3. Mobile Station sends a message MOB_MSHO_REQ which contains a list of all BSs.

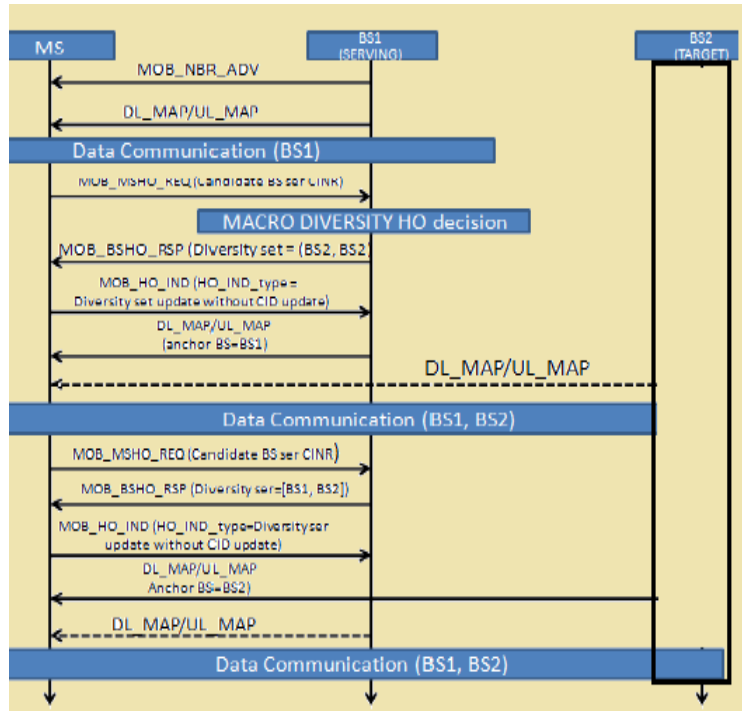

Fig.7. MAC Signaling for Dropping BS from Diversity Set

MS calculates RSSI, ranging and values of delay during propagation on the bases of MOB_NBR_ADV. In the response a message MOB_BSHO_RSP is received which contains a list of recommended base stations depending on Quality of Service or signal strength. Fig.6 represents the MAC signaling for connecting Base Station into diversity set. Fig.7 explains the messages used for disconnecting Base Station from diversity set.

\section{SIMULATIONS AND RESUlTS}

Simulation results explain three handover schemes. It also includes the results of comparison of Sector/site throughput $(\mathrm{Mb} / \mathrm{s})$ both uplink and downlink, and queuing delay with respect to WiMAX and WiMAX Release 2.

\section{A. Hard Handover Scheme}

In Hard Handover, firstly Mobile Station attached with Base Station 1. As it is "break before make" so, after 4 minutes of simulation time MS drops connection with BS1 due to low signal strength and connects with BS2 at the same time. Fig.8 and Fig.9 shows a throughput values. After 12 minutes MS breaks connection with BS2 and connects with BS3.

During Handover Process, Handover messages are exchanged. The spicks value in the graph represents handover messages. From the Fig. 8 and Fig.9 it is quite obvious that downlink throughput is similar to the received throughput of the BSs from backbone. Fig.10 
shows throughput sent by the BS and Fig.11 shows throughput received by BS.

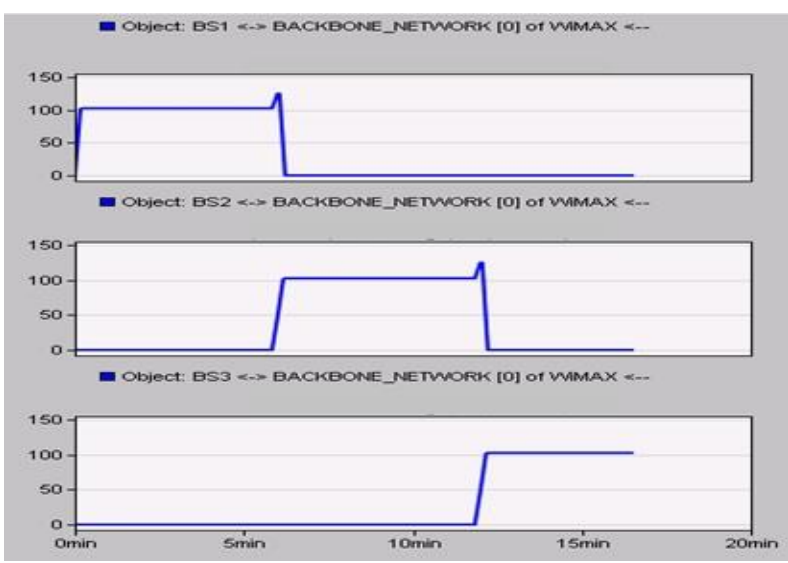

Fig.8. Downlink Throughput of MS

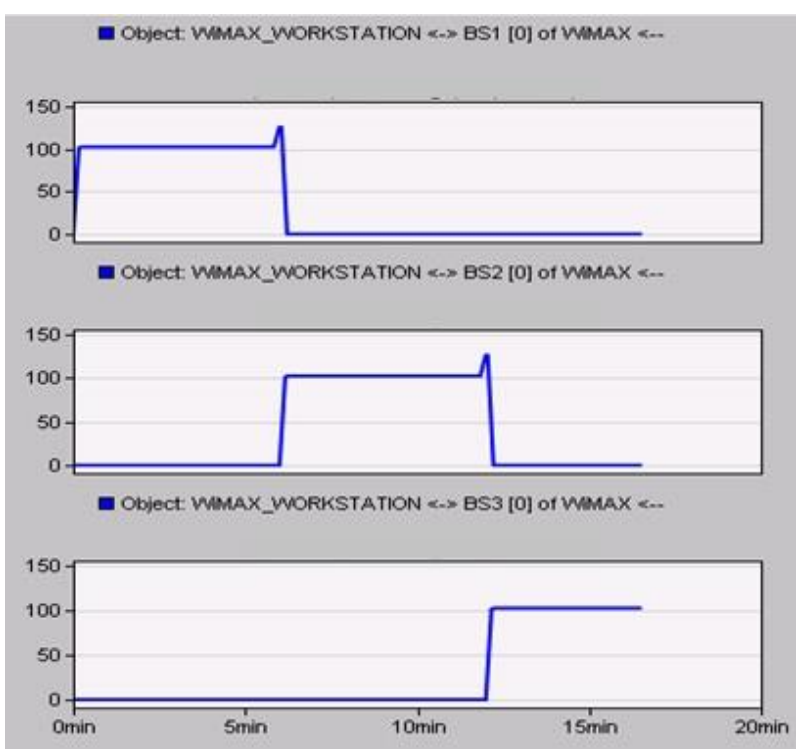

Fig.9. Uplink Throughput of MS

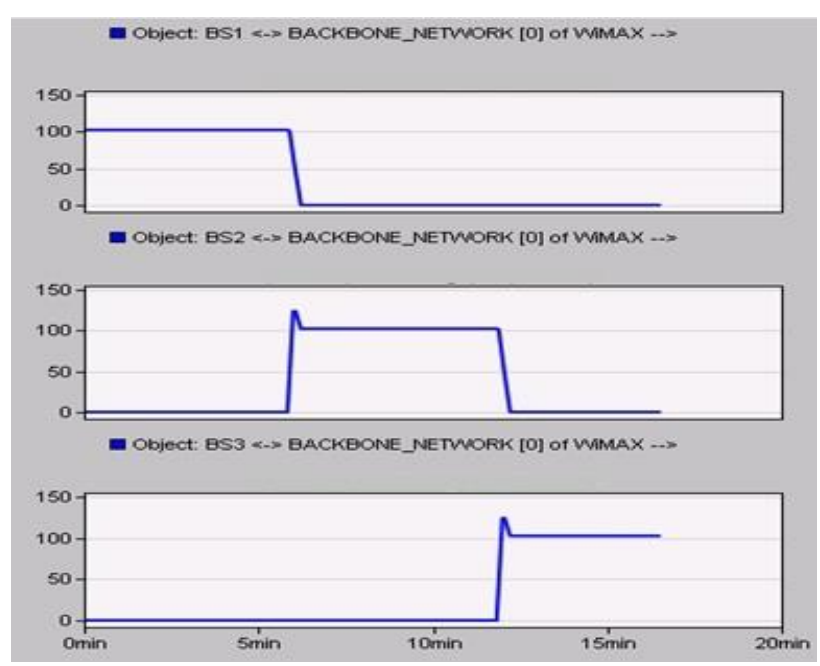

Fig.10. Throughput sent by BS 
become anchor and after 10 minutes Base Station 3 . Fig.14 shows point to point throughput of all BSs to uplink direction while Fig.15 shows uplink throughput from BSs to Backbone network. Figure 7.11 and Fig.12 shows point to point utilization to uplink and downlink directions respectively. Fig.12 and Fig.13 shows downlink and uplink queuing delay respectively.

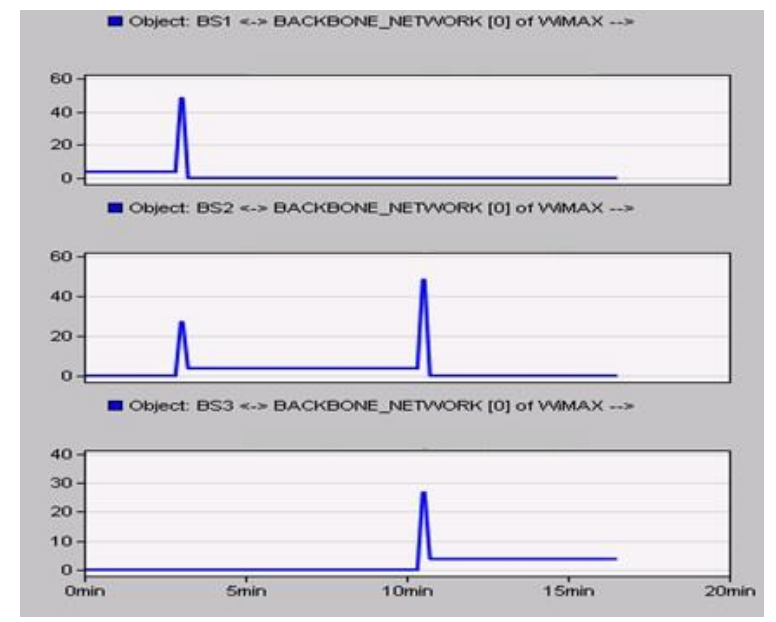

Fig.14. Uplink Throughput from BSs to Backbone

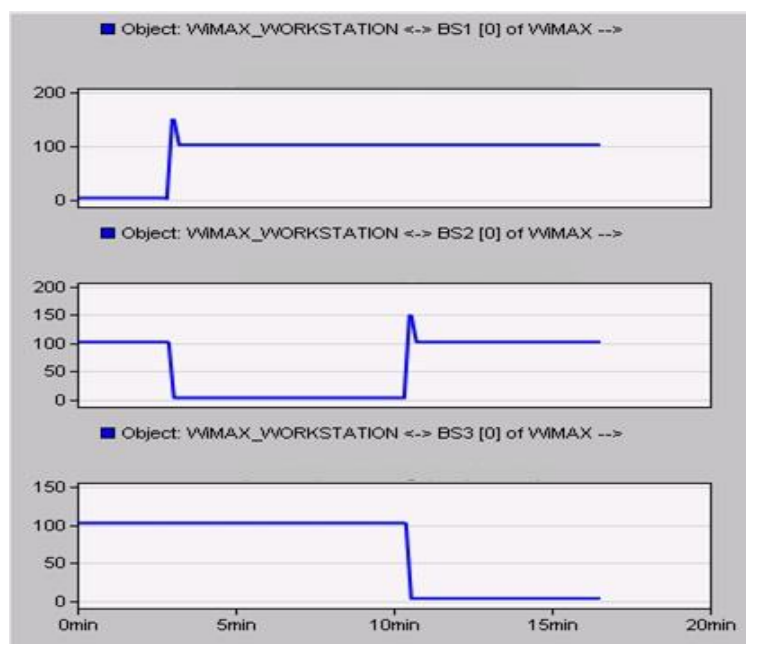

Fig.15. Uplink Throughput from MS to BSs

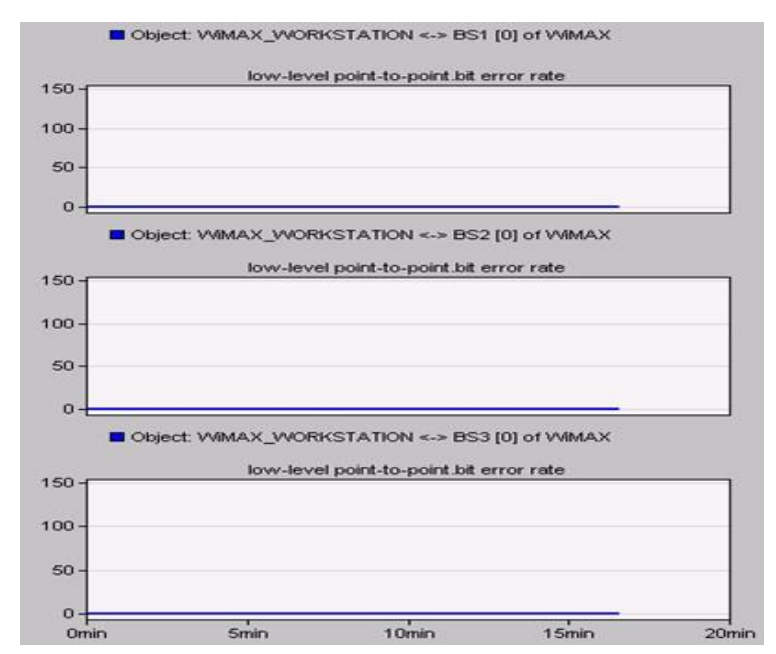

Fig.16. Bit Error Rate
Fig.16 shows the bit error rate for the different base stations.

\section{CONCLUSIONS}

This paper provides handover comparison between WiMAX and WiMAX Release 2. Due to high speed internet and enhanced application WiMAX Release 2 is the best and it's the beginning to $4 \mathrm{G}$ as well. WiMAX 2 provides bandwidth efficiency, lower latency and QoS enhancements, increased capacity for downlink and uplink VoIP, and multi-carrier support, increased sector/site throughput both at the uplink and downlink, voice capacity

\section{FUTURE WORK}

Technology and research have a strong relationship and both are directly related to engineers who are looking for more and more improvements including increased and reliable mobility, last end communication and enhanced QoS. Particularly the future work related to this paper can be enhanced by implementing the handover schemes of WiMAX 2 at MAC layer.

\section{REFERENCES}

[1] Augustine C. Odinma, P.D., Lawrence I. Oborkhale, M.Eng. and Muhammadou M.O. Kah, Ph.D., The Trends in Broadband Wireless Networks Technologies. The Pacific Journal of Science and Technology, 2007. 8(1): p. 7.

[2] Etemad, K., Overview of Mobile WiMAX Technology and Evolution. IEEE Communication Magazine, 2008. 46(10): p. 10.

[3] Carl Eklund, R.B.M., Kenneth L. Stanwood and Stanley Wang, IEEE Standard 802.16: A Technical Overview of the WirelessMAN Air Interface for Broadband Wireless Access. IEEE Communications Magazine, June 2002, 2002: p. 10.

[4] Doo Hwan, L., K. Kyamakya, and J.P. Umondi. Fast handover algorithm for IEEE 802.16e broadband wireless access system. in 2006 1st International Symposium on Wireless Pervasive Computing. 2006.

[5] S. Xu, M.M.a.C.H., Security Issues in Privacy and Key Management Protocol of IEEE 802.16. ACM Annual Southeast Regional conference, 2006. 44: p. 106.

[6] Ke, S.C., et al. A simplified ASN anchored mobility scheme over mobile WiMAX. in 2009 First Asian Himalayas International Conference on Internet. 2009.

[7] S. Choi, e.a., Fast handover scheme for real-time downlink services in IEEE 802.16e BWA systems. IEEEVehicular Technology Conference, 2005.

[8] Abdulrahman Yarali, S.R.a.B.M., WiMAX: 'The Innovative Broadband Wireless access technology. journals of communications, April, 2008. 3(2).

[9] Hamada, R.A., H.S. Ali, and M.I. Abdalla. An IMSbased LTE-WiMAX-WLAN architecture with efficient mobility management. in 2016 18th Mediterranean Electrotechnical Conference (MELECON). 2016.

[10] George, J. and P.P.E. Winston. Fast Mobile WiMAX handover by optimizing network topology acquisition phase procedure. in 2014 International Conference on 
Circuits, Power and Computing Technologies [ICCPCT-2014]. 2014.

[11] Ali Nawaz Khan, W.A., Ehsan Ullah Munir, Handover Techniques in Mobile WiMAX Networks:Analysis and Comparison. Middle-East Journal of Scientific Research, 2013: p. 7.

[12] Agrawal, A., A. Jeyakumar, and N. Pareek. Comparison between vertical handoff algorithms for heterogeneous wireless networks. in 2016 International Conference on Communication and Signal Processing (ICCSP). 2016.

[13] Chowdhury, A.S. and M.A. Gregory. UMTS and WiMAX handover performance comparison. in 2012 15th International Conference on Computer and Information Technology (ICCIT). 2012.

[14] Cheng-Wei Hu, Yung-Chung Wang, Jenn-Shing Wang,"Load Balancing Early Handoff Scheme for Multimedia Data Transmission in Wireless Networks", IJCNIS, vol.7, no.6, pp.1-9, 2015.DOI: 10.5815/ijcnis.2015.06.01

[15] Jyoti Madaan, Indu Kashyap,"Vertical Handoff Decision Algorithm Based on PRSS and Dwell Time", International Journal of Computer Network and Information Security(IJCNIS), Vol.8, No.7, pp.31-42, 2016.DOI: $10.5815 /$ ijcnis.2016.07.05

\section{Author's Profiles}

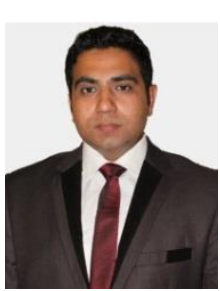

Ali Sufyan, has obtained his B.E. Telecommunication Engineering from IET, Rawalpindi, Pakistan in 2010 and M.Sc. in Personal Mobile and Satellite Communication from University of Bradford, Bradford, United Kingdom in 2011. Currently, He is working as Assistant Professor in Department of Electrical Engineering at GCUF (Government College University of
Faisalabad) Sahiwal, Pakistan. He has extensive knowledge of Telecommunication, Mobile and Satellite Communications. His research area includes but not limited to; WiMAX, Image Processing, Cloud, Renewable Energy and Wireless Networks.

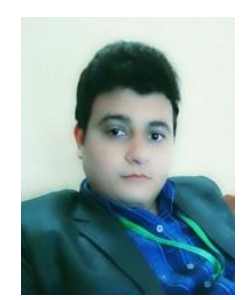

Yasir Salam obtained his B.S. Electrical Engineering with specialization in Telecommunication from GCUF, Faisalabad, Pakistan in 2013 and M.Sc. in Computer Engineering from University of Engineering and Technology, Lahore, Pakistan in 2016. Currently, He is working as Lecturer in Department of Computer Science at GCUF (Government College University of Faisalabad) Sahiwal, Pakistan. He has extensive knowledge of Telecommunication Networks His research areas are; Robotics, Multi-agent Systems, and Wireless Sensor Networks.

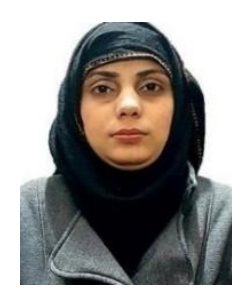

Sundas Amin has obtained her BS in Computer Science from Lahore College for Women University, Lahore, Pakistan in 2013 and MS in Computer Science from Lahore College for Women University, Lahore, Pakistan in 2015. She is currently working as a lecturer in Department of Computer Science at CIIT Sahiwal, Pakistan. She is very enthusiastic and having broad knowledge of Computer Science. Her research area includes Digital image Processing, Statistical Machine learning, Wireless and Mobile Communication (WiMAX).

How to cite this paper: Ali Sufyan, Yasir Salam, Sundas Amin,"Handover Comparisons of WiMAX and WiMAX Release 2", International Journal of Computer Network and Information Security(IJCNIS), Vol.9, No.8, pp.41-47, 2017.DOI: $10.5815 / \mathrm{ijcnis.2017.08.06}$ 